\title{
IMPROVED FRÉCHET BOUNDS AND MODEL-FREE PRICING OF MULTI-ASSET OPTIONS
}

\author{
PETER TANKOV, * École Polytechnique
}

\begin{abstract}
Improved bounds on the copula of a bivariate random vector are computed when partial information is available, such as the values of the copula on a given subset of $[0,1]^{2}$, or the value of a functional of the copula, monotone with respect to the concordance order. These results are then used to compute model-free bounds on the prices of two-asset options which make use of extra information about the dependence structure, such as the price of another two-asset option.
\end{abstract}

Keywords: Copula; Fréchet-Hoeffding bound; concordance order; basket option 2010 Mathematics Subject Classification: Primary 60E15; 91G20

\section{Introduction}

A (two-dimensional) copula is a function $C:[0,1]^{2} \rightarrow[0,1]$ with the following properties.

(i) Boundary conditions: $C(0, u)=C(u, 0)=0$ and $C(1, u)=C(u, 1)=u$ for all $u \in[0,1]$.

(ii) $C$ is 2 -increasing, i.e. for every $0 \leq u_{1}<u_{2} \leq 1$ and $0 \leq v_{1}<v_{2} \leq 1$, we have

$$
C\left(u_{2}, v_{2}\right)+C\left(u_{1}, v_{1}\right)-C\left(u_{1}, v_{2}\right)-C\left(u_{2}, v_{1}\right) \geq 0 .
$$

The classical Fréchet-Hoeffding bounds on the distribution function of a two-dimensional random vector can be expressed in terms of the copula $C$ of this vector:

$$
W(u, v):=\max \{0, u+v-1\} \leq C(u, v) \leq \min \{u, v\}:=M(u, v) .
$$

In the presence of additional information on the dependence between the components of the vector, these bounds can be narrowed. Nelsen et al. [10] computed the improved bounds when a measure of association, such as Kendall's $\tau$ or Spearman's $\rho$, is given, and the Bertino's family of copulas [2] yields best-possible bounds when the values of the copula on the main diagonal are known. More generally, given a nonempty set of bivariate copulas $\&$, Nelsen et al. [11] introduced pointwise best-possible bounds of $\$$ :

$$
A(u, v)=\sup \{C(u, v) \mid C \in \delta\} \quad \text { and } \quad B(u, v)=\inf \{C(u, v) \mid C \in \delta\} .
$$

These bounds are in general not copulas but quasi-copulas, and a fortiori they do not necessarily belong to the set $\&$.

Received 11 May 2010; revision received 8 February 2011.

* Postal address: Centre de Mathématiques Appliquées, École Polytechnique, 91128 Palaiseau, France.

Email address: peter.tankov@polytechnique.org 
In the theoretical part of this paper (Section 3), we first compute the improved Fréchet bounds when the values of the copula on an arbitrary subset of $[0,1]^{2}$ are given, and provide a sufficient condition for each bound to be a copula, and, therefore, be the best-possible bound. This generalizes the findings of [11] on the improved Fréchet bounds for copulas with given diagonal sections. Next, we compute the best-possible bounds when the value of a real-valued functional of the copula, monotone with respect to the concordance order and continuous with respect to pointwise convergence of copulas, is given, extending the results of [10].

Since the work of Rapuch and Roncalli [12], it has been known that the prices of most twoasset options, when the marginal laws of the two assets are fixed, become monotone functionals of the copula with respect to the concordance order. The classical Fréchet-Hoeffding bounds therefore lead to model-free price estimates for such options [1], [4], [5], [12].

In Section 4 we obtain a new representation for the price of a two-asset option, allowing us to use a quasi-copula. This representation enables us to compute (in Section 5) the improved model-free estimates of the option's value when the prices of all single-asset options on each of the two assets and some extra information about the dependence structure are known. This extra information may be, for example, the price of a different two-asset option (for example, zero-strike spread options are often quoted in the market), or the correlation of two assets. This is similar in spirit to a recent work by Kaas et al. [6], who computed worst-case bounds on the Value at Risk of a portfolio of two assets when the marginals and a measure of association are known.

\section{Preliminaries}

In this section we recall several useful definitions and results, and fix the notation for the rest of the paper. In the definition of quasi-copula [3], the 2-increasing property (1) is replaced by weaker assumptions.

Definition 1. A (two-dimensional) quasi-copula is a function $Q:[0,1]^{2} \rightarrow[0,1]$ with the following properties.

(i) $Q$ satisfies the boundary conditions: $Q(0, u)=Q(u, 0)=0$ and $Q(1, u)=Q(u, 1)=$ $u$ for all $u \in[0,1]$.

(ii) $Q$ is increasing in each argument.

(iii) $Q$ has the Lipschitz property: $\left|Q\left(u_{2}, v_{2}\right)-Q\left(u_{1}, v_{1}\right)\right| \leq\left|u_{2}-u_{1}\right|+\left|v_{2}-v_{1}\right|$ for all $\left(u_{1}, v_{1}, u_{2}, v_{2}\right) \in[0,1]^{4}$.

We denote the set of all copulas on $[0,1]^{2}$ by $\mathcal{C}$ and the set of all quasi-copulas by $\mathcal{Q}$. The concordance order is the order on $\mathcal{Q}$ defined by $Q_{1} \prec Q_{2}$ if and only if $Q_{1}(u) \leq Q_{2}(u)$ for all $u \in[0,1]^{2}$. It is clear that all quasi-copulas satisfy the Fréchet-Hoeffding bounds (2). Similarly, we say that $Q^{n} \rightarrow Q$ pointwise if $Q^{n}(u) \rightarrow Q(u)$ for all $u \in[0,1]^{2}$. The Lipschitz property implies that in this case the convergence is uniform in $u$.

For a copula or a quasi-copula $C$ and a rectangle $R=\left[u_{1}, u_{2}\right] \times\left[v_{1}, v_{2}\right] \subset[0,1]^{2}$, we define $V_{C}(R):=C\left(u_{2}, v_{2}\right)+C\left(u_{1}, v_{1}\right)-C\left(u_{1}, v_{2}\right)-C\left(u_{2}, v_{1}\right)$.

A subset $S \subset[0,1]^{2}$ is called increasing if, for all $\left(a_{1}, b_{1}\right) \in S$ and $\left(a_{2}, b_{2}\right) \in S$, either $a_{1} \leq a_{2}$ and $b_{1} \leq b_{2}$ or $a_{1} \geq a_{2}$ and $b_{1} \geq b_{2}$. It is called decreasing if, for all $\left(a_{1}, b_{1}\right) \in S$ and $\left(a_{2}, b_{2}\right) \in S$, either $a_{1} \leq a_{2}$ and $b_{1} \geq b_{2}$ or $a_{1} \geq a_{2}$ and $b_{1} \leq b_{2}$. It is easy to see that, for a decreasing set $S$, the set $\bar{S}:=\{(a, b):(a, 1-b) \in S\}$ is increasing. In the same spirit, if $C$ is 
a copula, the function $\bar{C}(u, v):=u-C(u, 1-v)$ is also a copula and if $Q$ is a quasi-copula, $\bar{Q}(u, v):=u-Q(u, 1-v)$ is also a quasi-copula.

The following well-known result (see, e.g. Theorem 3.2.3 of [9]) gives the best-possible bounds of a set of copulas taking a given value at a given point.

Proposition 1. Let $C$ be a copula, and suppose that $C(a, b)=\theta$ with $(a, b) \in[0,1]^{2}$. Then

$$
C_{L}^{a, b, \theta}(u, v) \leq C(u, v) \leq C_{U}^{a, b, \theta}(u, v), \quad(u, v) \in[0,1]^{2},
$$

where

and

$$
C_{U}^{a, b, \theta}=\min \left\{u, v, \theta+(u-a)^{+}+(v-b)^{+}\right\}
$$

$$
C_{L}^{a, b, \theta}=\max \left\{0, u+v-1, \theta-(a-u)^{+}-(b-v)^{+}\right\}
$$

are copulas satisfying $C_{U}^{a, b, \theta}(a, b)=C_{L}^{a, b, \theta}(a, b)=\theta$.

Remark 1. A careful examination of the proof or Theorem 3.2.3 of [9] reveals that (3) also holds if $C$ is a quasi-copula satisfying $C(a, b)=\theta$.

To close this section, we recall a well-known fact on distribution functions. Given a onedimensional distribution function $F(x)$, we define its generalized inverse by

$$
F^{-1}(u)=\inf \{x \in \mathbb{R}: F(x) \geq u\}, \quad u \in(0,1],
$$

with the convention that inf $\varnothing=+\infty$. If the couple $(X, Y)$ has copula $C$ then $(X, Y)$ has the same law as $\left(F_{X}^{-1}(U), F_{Y}^{-1}(V)\right)$, where $(U, V)$ are random variables with distribution function $C$.

\section{Constrained Fréchet bounds}

Let $S$ be a compact subset of $[0,1]^{2}$, and let $Q$ be a quasi-copula. We denote by $\mathcal{C}_{S}$ the set of all copulas $C^{\prime}$ such that $C^{\prime}(a, b)=Q(a, b)$ for all $(a, b) \in S$, and by $\mathcal{Q}_{S}$ the set of all quasi-copulas $Q^{\prime}$ such that $Q^{\prime}(a, b)=Q(a, b)$ for all $(a, b) \in S$. Define

$$
\begin{aligned}
& A^{S, Q}(u, v):=\min \left\{u, v, \min _{(a, b) \in S}\left\{Q(a, b)+(u-a)^{+}+(v-b)^{+}\right\}\right\}, \\
& B^{S, Q}(u, v):=\max \left\{0, u+v-1, \max _{(a, b) \in S}\left\{Q(a, b)-(a-u)^{+}-(b-v)^{+}\right\}\right\} .
\end{aligned}
$$

The following theorem establishes that $A^{S, Q}$ and $B^{S, Q}$ are best-possible bounds of the set $\mathcal{Q}_{S}$. This means that they are also bounds of the set $\mathcal{C}_{S}$, but not in general best possible. The second part of the theorem gives a sufficient condition under which $A^{S, Q}$ or $B^{S, Q}$ is a copula, and, therefore, a best-possible bound of $\mathcal{C}_{S}$. As a by-product of the second part, we obtain an example of a copula which coincides with a given quasi-copula on a given increasing or decreasing set.

Theorem 1. (i) $A^{S, Q}$ and $B S, Q$ are quasi-copulas satisfying

$$
B^{S, Q}(u, v) \leq Q^{\prime}(u, v) \leq A^{S, Q}(u, v) \text { for all }(u, v) \in[0,1]^{2}
$$

for every $Q^{\prime} \in Q_{S}$ and

$$
A^{S, Q}(a, b)=B^{S, Q}(a, b)=Q(a, b)
$$

for all $(a, b) \in S$. 
(ii) If the set $S$ is increasing then $B^{S, Q}$ is a copula; if the set $S$ is decreasing then $A^{S, Q}$ is a copula.

Proof. See Appendix A.

Example 1. This example, similar to Example 2.1 of [11], shows that if $S$ is increasing, $A^{S, Q}$ may not always be a copula. Let $S=\left\{\left(\frac{1}{3}, \frac{1}{3}\right),\left(\frac{2}{3}, \frac{2}{3}\right)\right\}$ and $Q=W$. Then

$$
A^{S, Q}\left(\frac{1}{3}, \frac{1}{3}\right)=0 \quad \text { and } \quad A^{S, Q}\left(\frac{2}{3}, \frac{2}{3}\right)=A^{S, Q}\left(\frac{1}{3}, \frac{2}{3}\right)=A^{S, Q}\left(\frac{2}{3}, \frac{1}{3}\right)=\frac{1}{3},
$$

so that the $A^{S, Q}$-volume of the rectangle $\left[\frac{1}{3}, \frac{2}{3}\right]^{2}$ is equal to $-\frac{1}{3}$. Similarly, if $S$ is decreasing, $B^{S, Q}$ is not always a copula.

Let $\rho: \mathcal{Q} \rightarrow \mathbb{R}$ be a mapping, continuous with respect to pointwise convergence of copulas and nondecreasing with respect to the concordance order on $\mathcal{Q}$. We are interested in computing pointwise best-possible bounds of the sets $\mathcal{C}^{r}:=\{C \in \mathcal{C}: \rho(C)=r\}$ and $\mathcal{Q}^{r}:=\{Q \in$ Q: $\rho(Q)=r$. We define

$$
\begin{gathered}
A^{r}(u, v):=\max \left\{C(u, v) \mid C \in \mathcal{C}^{r}\right\} \quad \text { and } \quad B^{r}(u, v):=\min \left\{C(u, v) \mid C \in \mathcal{C}^{r}\right\}, \\
\tilde{A}^{r}(u, v):=\max \left\{Q(u, v) \mid Q \in \mathcal{Q}^{r}\right\} \quad \text { and } \quad \tilde{B}^{r}(u, v):=\min \left\{Q(u, v) \mid Q \in \mathcal{Q}^{r}\right\},
\end{gathered}
$$

for $(u, v) \in[0,1]^{2}$.

For $(a, b) \in[0,1]^{2}$ and $\theta \in I_{a, b}:=[W(a, b), M(a, b)]$, we define

$$
\rho_{+}(a, b, \theta):=\rho\left(C_{U}^{a, b, \theta}\right), \quad \rho_{-}(a, b, \theta):=\rho\left(C_{L}^{a, b, \theta}\right) .
$$

For fixed $a$ and $b$, the mappings $\theta \mapsto \rho_{+}(a, b, \theta)$ and $\theta \mapsto \rho_{-}(a, b, \theta)$ are nondecreasing and continuous, and we define the corresponding inverse mappings by

$$
\begin{aligned}
r & \mapsto \rho_{-}^{-1}(a, b, r):=\max \left\{\theta \in I_{a, b}: \rho_{-}(a, b, \theta)=r\right\}, \\
r & \mapsto \rho_{+}^{-1}(a, b, r):=\min \left\{\theta \in I_{a, b}: \rho_{+}(a, b, \theta)=r\right\},
\end{aligned}
$$

for all $r$ such that the corresponding set over which the maximum or minimum is taken is nonempty.

Theorem 2. Let $r \in[\rho(W), \rho(M)]$. The bounds $A^{r}, \tilde{A}^{r}$ and $B^{r}, \tilde{B}^{r}$ are given by

$$
\begin{aligned}
& A^{r}(u, v)=\tilde{A}^{r}(u, v)= \begin{cases}\rho_{-}^{-1}(u, v, r) & \text { if } r \in\left[\rho(W), \rho_{-}(u, v, M(u, v))\right], \\
M(u, v) & \text { otherwise, }\end{cases} \\
& B^{r}(u, v)=\tilde{B}^{r}(u, v)= \begin{cases}\rho_{+}^{-1}(u, v, r) & \text { if } r \in\left[\rho_{+}(u, v, W(u, v)), \rho(M)\right], \\
W(u, v) & \text { otherwise. }\end{cases}
\end{aligned}
$$

Proof. See Appendix A.

Remark 2. Theorem 2 generalizes Theorems 2 and 4 of [10], which treat the cases when $\rho$ is Kendall's $\tau$ and Spearman's $\rho$. In these two cases, $A^{r}$ and $B^{r}$ are copulas. However, in general, this may not be the case. Let $\left(a_{1}, b_{1}\right) \in[0,1]^{2},\left(a_{2}, b_{2}\right) \in[0,1]^{2}, W\left(a_{1}, b_{1}\right) \leq \theta_{1} \leq$ $M\left(a_{1}, b_{1}\right)$, and $W\left(a_{2}, b_{2}\right) \leq \theta_{2} \leq M\left(a_{2}, b_{2}\right)$, and define

$$
\rho(C)=\left(C\left(a_{1}, b_{1}\right)-\theta_{1}\right)^{+}+\left(C\left(a_{2}, b_{2}\right)-\theta_{2}\right)^{+} .
$$


An easy computation shows that

$$
A^{0}(u, v)=\min \left\{u, v, \theta_{1}+\left(u-a_{1}\right)^{+}+\left(v-b_{1}\right)^{+}, \theta_{2}+\left(u-a_{2}\right)^{+}+\left(v-b_{2}\right)^{+}\right\},
$$

that is, we obtain the copula $A^{S, Q}$ of (4) with $S=\left\{\left(a_{1}, b_{1}\right),\left(a_{2}, b_{2}\right)\right\}$, and $Q$ such that $Q\left(a_{1}, b_{1}\right)=\theta_{1}$ and $Q\left(a_{2}, b_{2}\right)=\theta_{2}$. Then, Example 1 shows that $A^{0}$ is not always a copula.

\section{Copula-based pricing of multi-asset options}

We consider the problem of pricing a European-style option whose payoff depends on the values of two random variables $X$ and $Y$. These random variables can represent the terminal values of two assets (in the context of equity options) or some other risk factors which influence the value of the option, such as the default dates of two defaultable bonds.

We assume that the law of $X$ and $Y$ under the historical probability $\mathrm{P}$ is unknown, or is very hard to estimate, so that all information comes from the prices of traded options on these assets.

Under the standard assumption of absence of arbitrage opportunities in the market, the option pricing theory implies that there exists a risk-neutral probability $\mathrm{Q}$ such that the option price is given by the discounted expectation of its payoff under Q. In practice $\mathrm{Q}$ is not known, and only some incomplete information on it can be deduced from the prices of traded options on $X$ and $Y$.

We assume that these traded options include single-asset options, allowing us to reconstruct the cumulative distribution functions $F_{X}$ and $F_{Y}$ of $X$ and $Y$. For example, if $X$ is the price of an asset at time $T$ and call options on this asset with prices $P_{X}(K):=\mathrm{E}^{\mathrm{Q}}\left[\mathrm{e}^{-r T}(X-K)^{+}\right]$are available (where $r$ is the interest rate and $K$ is the strike price), the distribution function can be reconstructed as

$$
F_{X}(K)=1-\mathrm{e}^{r T} \frac{\partial P_{X}(K)}{\partial K} .
$$

Similarly, if $X$ is the default date of a defaultable bond, the distribution function may be reconstructed from the prices of credit default swaps on this bond with different maturities.

Let the discounted payoff function of a two-asset option be denoted by $f(x, y)$. Its price then becomes a function of the copula $C$ of $X$ and $Y$ :

$$
\begin{aligned}
\pi(C) & =\mathrm{E}^{\mathrm{Q}}[f(X, Y)] \\
& =\int_{0}^{\infty} \int_{0}^{\infty} f(x, y) \mathrm{d} C\left(F_{X}(x), F_{Y}(y)\right) \\
& =\int_{0}^{1} \int_{0}^{1} f\left(F_{X}^{-1}(u), F_{Y}^{-1}(v)\right) \mathrm{d} C(u, v) .
\end{aligned}
$$

It is known [8], [13] that, for every 2-increasing function $f$ such that the integral in (6) exists, the mapping $C \mapsto \pi(C)$ is nondecreasing with respect to the concordance order of copulas. Therefore, if the payoff function $f$ is 2-increasing, and if we know that the copula $C$ of $X$ and $Y$ satisfies $B \prec C \prec A$ for two copulas $A$ and $B$, the option price satisfies $\pi(B) \leq \pi(C) \leq \pi(A)$. For example, if no additional information on the joint law of $X$ and $Y$ is available, the standard Fréchet bounds lead to

$$
\int_{0}^{1} \int_{0}^{1} f\left(F_{X}^{-1}(u), F_{Y}^{-1}(v)\right) \mathrm{d} W(u, v) \leq \pi(C) \leq \int_{0}^{1} \int_{0}^{1} f\left(F_{X}^{-1}(u), F_{Y}^{-1}(v)\right) \mathrm{d} M(u, v) .
$$


Since the support of $\mathrm{d} M$ is the diagonal $v=u$ and that of $\mathrm{d} W$ is the diagonal $v=1-u$, these bounds are further simplified to

$$
\int_{0}^{1} f\left(F_{X}^{-1}(1-u), F_{Y}^{-1}(u)\right) \mathrm{d} u \leq \pi(C) \leq \int_{0}^{1} f\left(F_{X}^{-1}(u), F_{Y}^{-1}(u)\right) \mathrm{d} u .
$$

However, if $A$ and $B$ are quasi-copulas, this method no longer applies because the integral in (6) may not be well defined. The following result provides an alternative representation for $\pi(C)$ which can be used for quasi-copulas, and establishes other useful properties of this mapping. We recall [7, Section 4.5] that, for a 2-increasing function $f$ on $[0, \infty)^{2}$ which is left continuous in both arguments, there exists a unique positive measure $\mu$ on $[0, \infty)^{2}$ such that

$$
\mu\left(\left[x_{1}, x_{2}\right) \times\left[y_{1}, y_{2}\right)\right)=f\left(x_{1}, y_{1}\right)+f\left(x_{2}, y_{2}\right)-f\left(x_{1}, y_{2}\right)-f\left(x_{2}, y_{1}\right) .
$$

Proposition 2. Assume that $f$ is 2-increasing, left continuous in each of its arguments, and let the marginal laws of $X$ and $Y$ satisfy

$$
\mathrm{E}[|f(X, 0)|+|f(0, X)|+|f(Y, 0)|+|f(0, Y)|+|f(X, X)|+|f(Y, Y)|]<\infty .
$$

Then, $\mathrm{E}[|f(X, Y)|]<\infty$ and the mapping $C \mapsto \pi(C)$ is well defined for all $C$, continuous with respect to pointwise convergence of copulas, and satisfies

$$
\begin{aligned}
\pi(C)= & -f(0,0)+\mathrm{E}[f(X, 0)]+\mathrm{E}[f(0, Y)] \\
& +\int_{0}^{\infty} \int_{0}^{\infty} \mu(\mathrm{d} x \times \mathrm{d} y)\left(1-F_{X}(x)-F_{Y}(y)+C\left(F_{X}(x), F_{Y}(Y)\right)\right),
\end{aligned}
$$

where $\mu$ is the positive measure on $[0, \infty)^{2}$ induced by $f$.

Proof. See Appendix A.

Remark 3. Expression (8) can be alternatively written as

$$
\pi(C)=-f(0,0)+\mathrm{E}[f(X, 0)]+\mathrm{E}[f(0, Y)]+\int_{0}^{\infty} \int_{0}^{\infty} \mu(\mathrm{d} x \times \mathrm{d} y) \bar{C}\left(\bar{F}_{X}(x), \bar{F}_{Y}(Y)\right),
$$

where $\bar{C}$ is the survival copula defined by

$$
\bar{C}(u, v)=u+v-1+C(1-u, 1-v),
$$

and $\bar{F}_{X}$ and $\bar{F}_{Y}$ are survival functions of $X$ and $Y$.

Table 1 gives several examples of two-asset options whose payoffs are 2-increasing (or 2decreasing, meaning that $-f$ is 2 -increasing) continuous functions. These are mainly taken from [12]. For all these payoffs, the integral with respect to $\mu$ in (8) reduces to a one-dimensional integral. Another important example is the function $f(X, Y)=X Y$, which is also 2-increasing, which means that, for fixed marginal distributions, the linear correlation coefficient

$$
\rho(X, Y)=\frac{\mathrm{E}[X Y]-\mathrm{E}[X] \mathrm{E}[Y]}{(\operatorname{var} X \operatorname{var} Y)^{1 / 2}}
$$

is nondecreasing with respect to the concordance order of copulas. The corresponding measure $\mu$ is the Lebesgue measure on $[0, \infty)^{2}$. 
TABLE 1: Common two-asset option payoff functions, and the representation of integrals with respect to the corresponding measure $\mu$. The plus sign indicates that the payoff function is 2-increasing and the minus sign indicates that it is 2-decreasing.

\begin{tabular}{|c|c|c|}
\hline Option type and $f(X, Y)$ & Increasing? & $\int_{0}^{\infty} \int_{0}^{\infty} \mu(\mathrm{d} x \times \mathrm{d} y) G(x, y)$ \\
\hline $\begin{array}{l}\text { Basket option, } \\
\qquad(\alpha X+\beta Y-K)^{+}\end{array}$ & $\begin{array}{l}+ \text { if } \alpha \beta>0 \\
- \text { if } \alpha \beta<0\end{array}$ & $\operatorname{sgn}(\alpha \beta) \int_{\{z: z / \alpha>0,(K-z) / \beta>0\}} G(z / \alpha,(K-z) / \beta) \mathrm{d} z$ \\
\hline $\begin{array}{l}\text { Call on the minimum } \\
\qquad(\min \{X, Y\}-K)^{+}\end{array}$ & + & $\int_{K}^{\infty} G(x, x) \mathrm{d} x$ \\
\hline $\begin{array}{l}\text { Put on the minimum } \\
\qquad(K-\min \{X, Y\})^{+}\end{array}$ & + & $\int_{0}^{K} G(x, x) \mathrm{d} x$ \\
\hline $\begin{array}{l}\text { Call on the maximum } \\
\qquad(\max \{X, Y\}-K)^{+}\end{array}$ & - & $-\int_{K}^{\infty} G(x, x) \mathrm{d} x$ \\
\hline $\begin{array}{l}\text { Put on the maximum } \\
\qquad(K-\max \{X, Y\})^{+}\end{array}$ & - & $-\int_{0}^{K} G(x, x) \mathrm{d} x$ \\
\hline $\begin{array}{l}\text { Worst-off call } \\
\quad \min \left\{\left(X-K_{1}\right)^{+},\left(Y-K_{2}\right)^{+}\right\} \\
\text {Worst-off put }\end{array}$ & + & $\int_{0}^{\infty} G\left(z+K_{1}, z+K_{2}\right) \mathrm{d} z$ \\
\hline $\begin{array}{l}\min \left\{\left(K_{1}-X\right)^{+},\left(K_{2}-Y\right)^{+}\right\} \\
\text {Best-off call }\end{array}$ & + & $\int_{0}^{\min \left\{K_{1}, K_{2}\right\}} G\left(K_{1}-z, K_{2}-z\right) \mathrm{d} z$ \\
\hline $\begin{array}{l}\quad \max \left\{\left(X-K_{1}\right)^{+},\left(Y-K_{2}\right)^{+}\right\} \\
\text {Best-off put }\end{array}$ & - & $-\int_{0}^{\infty} G\left(z+K_{1}, z+K_{2}\right) \mathrm{d} z$ \\
\hline $\max \left\{\left(K_{1}-X\right)^{+},\left(K_{2}-Y\right)^{+}\right\}$ & - & $-\int_{0}^{\min \left\{K_{1}, K_{2}\right\}} G\left(K_{1}-z, K_{2}-z\right) \mathrm{d} z$ \\
\hline
\end{tabular}

\section{Application: model-free bounds on option prices}

In this section we derive model-free bounds on the prices of two-asset options whose payoff function satisfies the assumptions of Proposition 2 when extra information about the dependence of $X$ and $Y$ is given. We give four examples corresponding to different kinds of extra information and different option payoffs.

Example 2. (The case when prices of digital basket options are known.) As in our first example, we consider an application to credit risk modeling, assuming that $X$ and $Y$ represent the times of default of two corporate bonds. In this context, an important problem is the pricing of the so-called 'first to default' option with payoff at maturity $T$ given by $H_{T}^{1}=\mathbf{1}_{\{(X \wedge Y) \leq T\}}$ or the 'second to default' option with payoff $H_{T}^{2}=\mathbf{1}_{\{(X \vee Y) \leq T\}}$. The price of each of these options is directly related to the value of the copula $C$ of $X$ and $Y$ at the point $\left(F_{X}(T), F_{Y}(T)\right)$ :

$$
\begin{aligned}
& \mathrm{E}^{\mathrm{Q}}\left[H_{T}^{1}\right]=1-\mathrm{P}[X>T, Y>T]=F_{X}(T)+F_{Y}(T)-C\left(F_{X}(T), F_{Y}(T)\right), \\
& \mathrm{E}^{\mathrm{Q}}\left[H_{T}^{2}\right]=\mathrm{P}[X \leq T, Y \leq T]=C\left(F_{X}(T), F_{Y}(T)\right) .
\end{aligned}
$$

In view of the above, we concentrate on the 'second to default' options. From the prices $P_{k}=\mathrm{E}\left[H_{T_{k}}^{2}\right]$ of these options with maturities $T_{1}, \ldots, T_{n}$, we can recover the values of the copula $C$ of $X$ and $Y$ on the increasing set $\left(F_{X}\left(T_{k}\right), F_{Y}\left(T_{k}\right)\right)_{k=1, \ldots, n}$. Therefore, by Theorem 1 , the copula $C$ of $X$ and $Y$ satisfies

$$
B(u, v) \leq C(u, v) \leq A(u, v) \text { for all }(u, v) \in[0,1]^{2}
$$




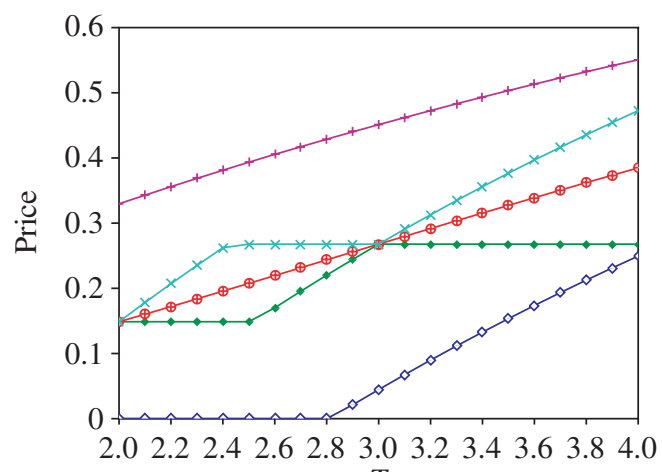

T

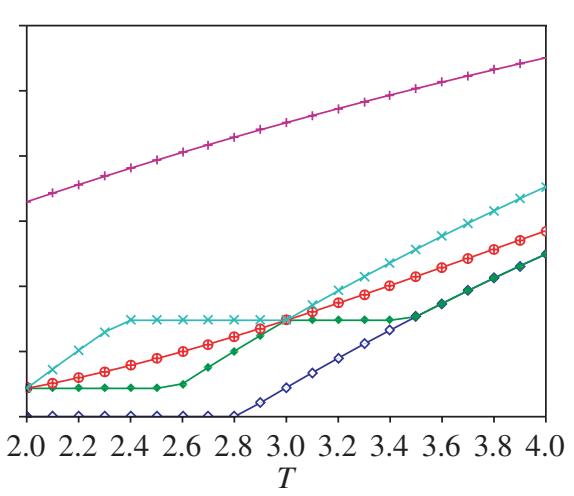

$\leftrightarrow$ Lower Fréchet $\leftrightarrow$ Lower improved $\leftrightarrow$ Gaussian copula

$x \times x$ Upper improved $\longrightarrow$ Upper Fréchet

FIGURE 1: Improved bound on the prices of 'second to default' options as a function of time to maturity $T$, when the prices of options with two and three years to maturity are known and equal to the price in the

'Gaussian copula' model with given correlation $\rho$. Left: $\rho=0$. Right: $\rho=-0.7$.

with

$$
\begin{aligned}
& A(u, v)=\min \left\{u, v, \min _{k=1, \ldots, n}\left\{P_{k}+\left(u-F_{X}\left(T_{k}\right)\right)^{+}+\left(v-F_{Y}\left(T_{k}\right)\right)^{+}\right\}\right\}, \\
& B(u, v)=\max \left\{0, u+v-1, \max _{k=1, \ldots, n}\left\{P_{k}-\left(F_{X}\left(T_{k}\right)-u\right)^{+}-\left(F_{Y}\left(T_{k}\right)-y\right)^{+}\right\}\right\} .
\end{aligned}
$$

The price of any two-asset option whose payoff function $f(x, y)$ satisfies the assumption of Proposition 2 admits the bounds

$$
\pi(B) \leq \pi(C) \leq \pi(A)
$$

Since, by Theorem $1, B$ is a copula, the lower bound is sharp, while the upper bound may not necessarily be sharp.

As an illustration, we have computed the upper and lower improved bounds for the prices of 'second to default' options with different times to maturity. We assume that the marginal laws of $X$ and $Y$ are exponential with parameters $\lambda_{X}=0.2$ and $\lambda_{Y}=0.3$, respectively, and that the prices of the 'second to default' options with two and three years to maturity are known. In this example, these two prices are computed assuming that $X$ and $Y$ have Gaussian copula with correlation $\rho$ (the Gaussian copula is the industry standard). Figure 1 shows the prices of the 'second to default' options as a function of the time to maturity for two different values of $\rho$, along with the price in the 'Gaussian copula' model and the standard Fréchet bounds (without any information about dependence).

Example 3. (The case when prices of all options on the maximum of two assets are known.) The knowledge of prices of call or put options on the maximum or the minimum of $X$ and $Y$, for all strikes, allows us to recover (by differentiation) the values of the distribution function $F(K, K)$ for $K \geq 0$, or, equivalently, the values of the copula $C$ on the increasing set $\left(\left(F_{X}(K), F_{Y}(K)\right), K \geq 0\right)$. Therefore, similarly to the previous example, the copula $C$ of 

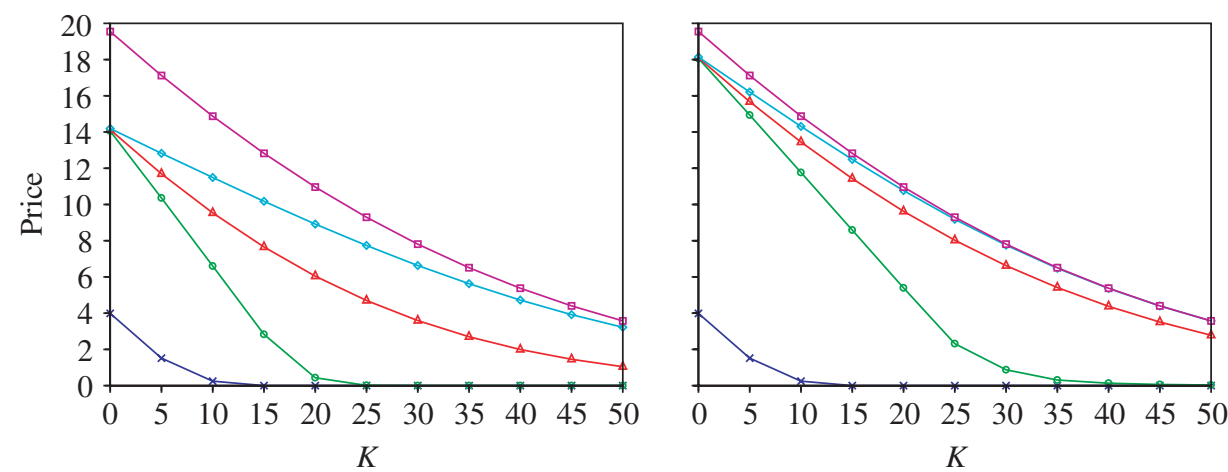

Lower Fréchet

$\leftrightarrow$ Lower improved

$\triangle-\Delta-\Delta$ BS price

$\leftrightarrow$ Upper improved

$\rightarrow-$ Upper Fréchet

FIGURE 2: Improved bounds on the spread option price as a function of the strike $K$, when the prices of all options on the maximum are known and equal to the price in the Black-Scholes (BS) model with given correlation $\rho$. Left: $\rho=0$. Right: $\rho=-0.7$.

$X$ and $Y$ satisfies

$$
B(u, v) \leq C(u, v) \leq A(u, v) \text { for all }(u, v) \in[0,1]^{2},
$$

where

$$
\begin{aligned}
& A(u, v)=\min \left\{u, v, \min _{K \geq 0}\left\{F(K, K)+\left(u-F_{X}(K)\right)^{+}+\left(v-F_{Y}(K)\right)^{+}\right\}\right\}, \\
& B(u, v)=\max \left\{0, u+v-1, \max _{K \geq 0}\left\{F(K, K)-\left(F_{X}(K)-u\right)^{+}-\left(F_{Y}(K)-y\right)^{+}\right\}\right\} .
\end{aligned}
$$

To illustrate this method, we have computed the improved upper and lower bounds for the spread option with payoff at date $T=1$ given by $f\left(X_{T}, Y_{T}\right)=\left(X_{T}-Y_{T}-K\right)^{+}$. To fix the marginal laws of $X$ and $Y$, we assume that $X_{t}=X_{0} \exp \left(\sigma_{x} W_{t}^{x}-\sigma_{x}^{2} t / 2\right)$ and $Y_{t}=Y_{0} \exp \left(\sigma_{y} W_{t}^{y}-\sigma_{y}^{2} t / 2\right)$, where $\sigma_{x}=0.2, \sigma_{y}=0.3, X_{0}=Y_{0}=100$, and $W^{y}$ and $W^{x}$ are standard Brownian motions. We further assume that the prices of all options on the maximum of $X$ and $Y$ are equal to the corresponding prices in a model where $W_{T}^{y}$ and $W_{T}^{x}$ are jointly Gaussian with correlation $\rho$.

In Figure 2 we plot the improved bounds on the spread option price as a function of the strike $K$ for two different values of the correlation $\rho$, along with the Black-Scholes price and the standard Fréchet bounds. For the numerical computation of the bounds, we have taken a discrete set of 400 strikes in (9) and (10), and used numerical integration to evaluate (8), which reduces to a one-dimensional integral in this case.

Example 4. (The case when a single option price is known.) Assume now that the extra information about the dependence structure of $X$ and $Y$ is the expectation of a function $f_{0}$ which satisfies the assumptions of Proposition 2: $\rho(C):=\mathrm{E}^{\mathrm{Q}}\left[f_{0}(X, Y)\right]=r$. In this case, the price of a two-asset option whose payoff $f(x, y)$ satisfies the assumptions of Proposition 2 admits the bounds $\pi\left(B^{r}\right) \leq \pi(C) \leq \pi\left(A^{r}\right)$ with $A^{r}$ and $B^{r}$ given by Theorem 2. Although $A^{r}$ and $B^{r}$ are best-possible bounds of the set of copulas satisfying $\rho(C)=r$, if they are not copulas themselves, the bounds on the option price may not be best possible. 

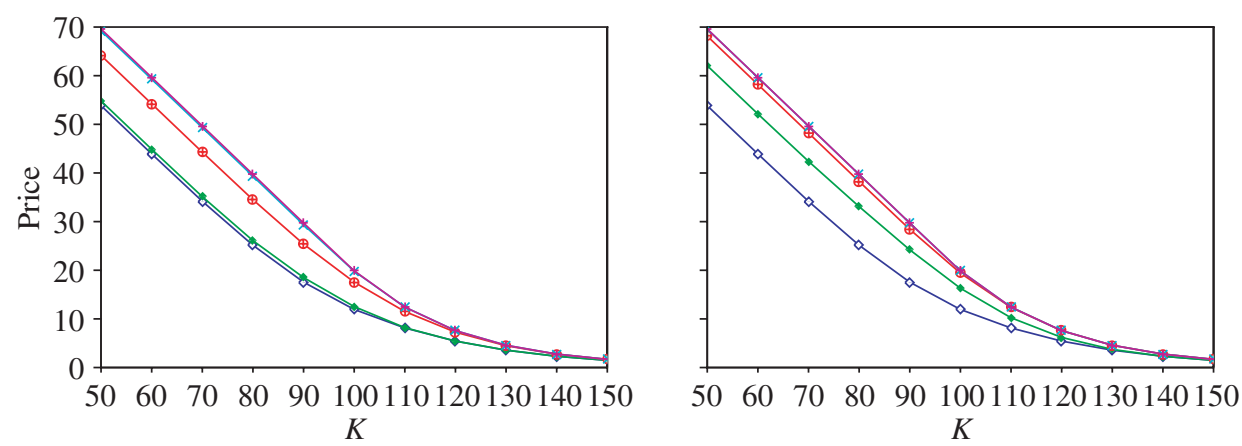

$\leftrightarrow$ Lower Fréchet $\leftrightarrow$ Lower improved $\leftrightarrow$ BS price

$\star * x$ Upper improved $\longleftrightarrow$ Upper Fréchet

FIGURE 3: Improved bounds on the price of the option on the maximum of two assets as a function of the strike $K$ when the price of the zero-strike spread option is known and equal to the price in the Black-Scholes (BS) model with given correlation $\rho$. Left: $\rho=0$. Right: $\rho=-0.7$.

For the actual computation of $A^{r}$ and $B^{r}$, we reduce the expressions for $\rho^{+}(a, b, \theta)$ and $\rho^{-}(a, b, \theta)$ to one-dimensional integrals using the results of [9, Section 3.2.3]:

$$
\begin{aligned}
\rho^{+}(a, b, \theta)= & \int_{0}^{\theta} f_{0}\left(F_{X}^{-1}(u), F_{Y}^{-1}(u)\right) \mathrm{d} u+\int_{\theta}^{a} f_{0}\left(F_{X}^{-1}(u), F_{Y}^{-1}(u+b-\theta)\right) \mathrm{d} u \\
& +\int_{a}^{a+b-\theta} f_{0}\left(F_{X}^{-1}(u), F_{Y}^{-1}(u+\theta-a)\right) \mathrm{d} u \\
& +\int_{a+b-\theta}^{1} f_{0}\left(F_{X}^{-1}(u), F_{Y}^{-1}(u)\right) \mathrm{d} u, \\
\rho^{-}(a, b, \theta)= & \int_{0}^{a-\theta} f_{0}\left(F_{X}^{-1}(u), F_{Y}^{-1}(1-u)\right) \mathrm{d} u \\
& +\int_{a-\theta}^{a} f_{0}\left(F_{X}^{-1}(u), F_{Y}^{-1}(a+b-\theta-u)\right) \mathrm{d} u \\
& +\int_{a}^{1-b+\theta} f_{0}\left(F_{X}^{-1}(u), F_{Y}^{-1}(1+\theta-u)\right) \mathrm{d} u \\
& +\int_{1-b+\theta}^{1} f_{0}\left(F_{X}^{-1}(u), F_{Y}^{-1}(1-u)\right) \mathrm{d} u .
\end{aligned}
$$

As the first illustration of this approach, we have computed the improved bounds on the price of the call option on the maximum of two assets, with payoff at date $T=1$ given by $f\left(X_{T}, Y_{T}\right)=\left(\max \left\{X_{T}, Y_{T}\right\}-K\right)^{+}$, assuming that the price of the zero-strike spread option, with payoff $f_{0}\left(X_{T}, Y_{T}\right)=\left(X_{T}-Y_{T}\right)^{+}$, is known (these options are indeed often quoted in the market). The marginal laws of $X$ and $Y$ are the same as in Example 3, and we further assume that the price of the zero-strike spread option is equal to the corresponding price in a model where $W_{T}^{y}$ and $W_{T}^{x}$ are jointly Gaussian with correlation $\rho$.

In Figure 3 we plot the improved bounds as a function of the strike $K$ for two different values of the correlation $\rho$, along with the Black-Scholes price and the standard Fréchet bounds. Since 


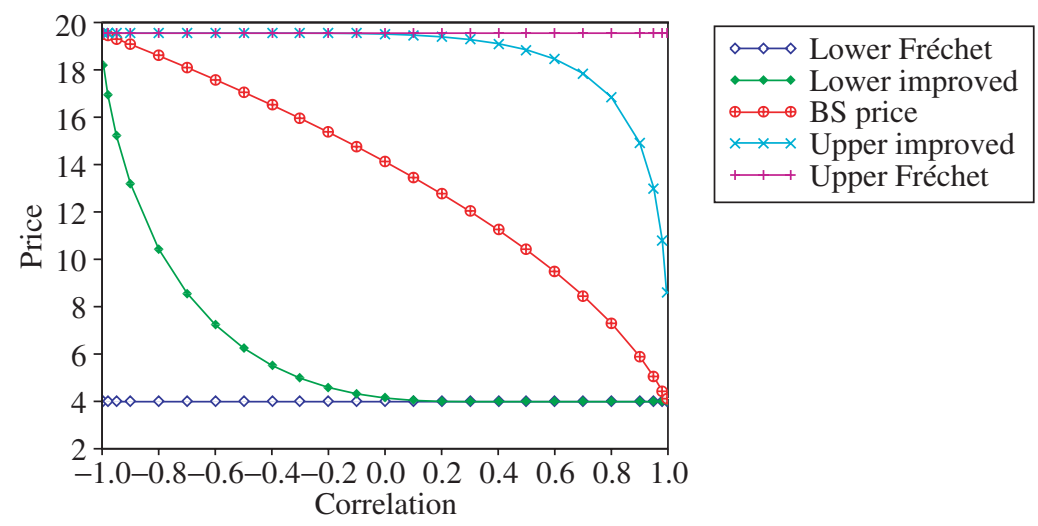

FIGURE 4: Improved bounds on the price of the zero-strike spread option when the correlation of logreturns $\rho$ is known, as a function of $\rho$. For comparison, the price in the Black-Scholes model with the given value of correlation is shown as the BS price.

we now have much less information on the dependence of $X_{T}$ and $Y_{T}$ than in Example 3, the improved bounds are not as narrow as in that example. Still, when the spread option price is close to one of its extreme values, such as, for example in the right-hand diagram of Figure 3, where we have taken $\rho=-0.7$, the improved bounds lead to a considerable narrowing of the price interval. In the numerical example, $\rho^{+}$and $\rho^{-}$were evaluated by numerical integration, their inverses were then computed by bisection, and a further numerical integration was performed to evaluate the bounds.

Example 5. (The case when the linear correlation of log-returns is known.) Very often, the option trader does not know the full two-dimensional distribution of $X$ and $Y$ under $\mathrm{Q}$, but has a strong view about the risk-neutral correlation of log-returns

$$
\rho_{0}=\frac{\mathrm{E}[\log X \log Y]-\mathrm{E}[\log X] \mathrm{E}[\log Y]}{(\operatorname{var}(\log X) \operatorname{var}(\log Y))^{1 / 2}} .
$$

In this case, we can obtain bounds on the prices of two-asset options in the same way as in Example 4, using the function $f_{0}(x, y)=\log x \log y$, which is 2-increasing. In Figure 4 we plot the bounds on the price of a zero-strike spread option with payoff $f\left(X_{T}, Y_{T}\right)=\left(X_{T}-Y_{T}\right)^{+}$ when the correlation of log-returns is known, for different correlation values. As we have already observed in Example 4, these bounds are most useful for extreme correlation scenarios, and yield little additional information when the correlation is close to 0 .

\section{Appendix A. The proofs}

Proof of Theorem 1. First, observe that $A$ can be obtained from $B$ by a simple transformation:

$$
A^{S, Q}(u, v)=u-B^{\bar{S}, \bar{Q}}(u, 1-v)=\bar{B}^{\bar{S}, \bar{Q}}(u, v)
$$

(the bar notation was introduced in Section 2). It is therefore sufficient to prove only the statements involving $B$ S, . 
(i) Let us first check that $B S, Q$ is a quasi-copula. The boundary conditions follow from the Fréchet bounds for $Q$. The fact that $B S, Q$ is increasing in each argument is obvious, and the Lipschitz property follows because, for a family of functions $\left(f_{i}\right)_{i \in I}$ which are Lipschitz with constant 1 , we have

$$
\max _{i} f_{i}(y) \leq|x-y|+\max _{i} f_{i}(x) \text { and } \max _{i} f_{i}(x) \leq|x-y|+\max _{i} f_{i}(y),
$$

which implies that $\max _{i} f_{i}(x)$ is Lipschitz with the same constant. By Proposition 1 and Remark $1, C_{L}^{a, b, Q(a, b)}(u, v) \leq Q^{\prime}(u, v)$ for all $(u, v) \in[0,1]^{2},(a, b) \in S$, and $Q^{\prime} \in Q_{S}$. Since $B^{S, Q}$ is the upper bound of $C_{L}^{a, b, Q(a, b)}(u, v)$ over $(a, b) \in S$, we have $B^{S, Q}(u, v) \leq Q^{\prime}(u, v)$.

Let us now check property (5). Take $\left(a^{\prime}, b^{\prime}\right) \in S$. From the Fréchet lower bound for $Q$, we obtain

$$
B^{S, Q}\left(a^{\prime}, b^{\prime}\right)=\max _{(a, b) \in S}\left\{Q(a, b)-\left(a-a^{\prime}\right)^{+}-\left(b-b^{\prime}\right)^{+}\right\} .
$$

For every $(a, b) \in S$, using the Lipschitz property of $Q$ and the fact that it is increasing in each argument, we obtain

$$
Q(a, b)-\left(a-a^{\prime}\right)^{+}-\left(b-b^{\prime}\right)^{+} \leq Q\left(a^{\prime}, b^{\prime}\right) .
$$

Therefore, the max is attained for $(a, b)=\left(a^{\prime}, b^{\prime}\right)$.

(ii) Let $S$ be an increasing set. By adding to this set the points $(0,0)$ and $(1,1)$, we may without loss of generality simplify the definition of $B^{S, Q}$ :

$$
B^{S, Q}(u, v):=\max _{(a, b) \in S}\left\{Q(a, b)-(a-u)^{+}-(b-v)^{+}\right\} .
$$

Given that $B S, Q$ is a quasi-copula, we only need to prove property (1).

Since $B^{S, Q}$ is Lipschitz continuous, for every $\varepsilon>0$, we can find a finite increasing set $S_{\varepsilon}$ such that $\sup _{(u, v) \in[0,1]^{2}}\left|B^{S_{\varepsilon}, Q}(u, v)-B^{S, Q}(u, v)\right| \leq \varepsilon$. Therefore, it is enough to prove property (1) for a set $S_{n}=\left\{\left(a_{i}, b_{i}\right)\right\}_{i=1}^{n}$, where we suppose without loss of generality that $a_{i} \leq a_{i+1}$ and $b_{i} \leq b_{i+1}$ for $i=1, \ldots, n-1$.

The proof will be done by induction. For $n=1$, property (1) is straightforward. Assume that it holds for $S_{n}$, and let $a_{n+1} \geq a_{n}, b_{n+1} \geq b_{n}$, and $S_{n+1}:=S_{n} \cup\left\{\left(a_{n+1}, b_{n+1}\right)\right\}$. To simplify the notation, we write $B_{n}:=B^{S_{n}, Q}, B_{n+1}:=B^{S_{n+1}, Q}$, and $Q_{n+1}:=Q\left(a_{n+1}, b_{n+1}\right)$. For convenience, we subdivide the domain $[0,1]^{2}$ into four sets $A, B, C$, and $D$ as shown in Figure 5 .

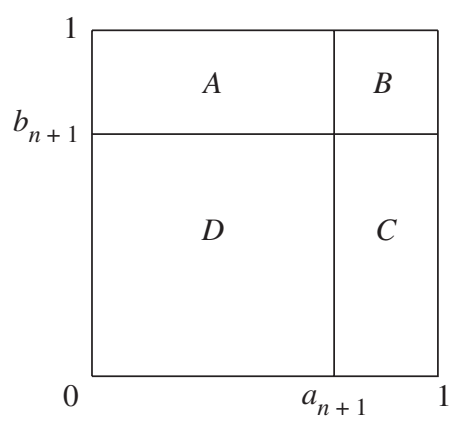

FIGURE 5: Illustration for the proof of Theorem 1(ii). 
To prove that $B_{n+1}$ is 2 -increasing, we must show that, for every rectangle $R \subset[0,1]^{2}$, $V_{B_{n+1}}(R) \geq 0$. However, since $V_{B}$ is additive over rectangles, it is sufficient to consider only the cases $R \subseteq A, R \subseteq B, R \subseteq C$, and $R \subseteq D$. By construction, on $A$, the function $B_{n+1}$ depends only on the coordinate $u$, and, therefore, $V_{B_{n+1}}(R)=0$ for every rectangle $R \subseteq A$. Similarly, $V_{B_{n+1}}(R)=0$ for $R \subseteq B$ because $B_{n+1}$ is constant on $B$ and $V_{B_{n+1}}(R)=0$ for $R \subseteq C$ because $B_{n+1}$ depends only on the coordinate $v$ on $C$. It remains to consider the case $R \subseteq D$.

Let $R=\left[u_{1}, u_{2}\right] \times\left[v_{1}, v_{2}\right] \subseteq D$. We must show that

$$
\begin{aligned}
V_{B_{n+1}}(R)= & \max \left\{B_{n}\left(u_{1}, v_{1}\right), Q_{n+1}-\left(a_{n+1}-u_{1}\right)-\left(b_{n+1}-v_{1}\right)\right\} \\
& +\max \left\{B_{n}\left(u_{2}, v_{2}\right), Q_{n+1}-\left(a_{n+1}-u_{2}\right)-\left(b_{n+1}-v_{2}\right)\right\} \\
& -\max \left\{B_{n}\left(u_{1}, v_{2}\right), Q_{n+1}-\left(a_{n+1}-u_{1}\right)-\left(b_{n+1}-v_{2}\right)\right\} \\
& -\max \left\{B_{n}\left(u_{2}, v_{1}\right), Q_{n+1}-\left(a_{n+1}-u_{2}\right)-\left(b_{n+1}-v_{1}\right)\right\} \\
\geq & 0 .
\end{aligned}
$$

We consider three cases separately.

- If $B_{n}\left(u_{1}, v_{2}\right) \geq Q_{n+1}-\left(a_{n+1}-u_{1}\right)-\left(b_{n+1}-v_{2}\right)$ and $B_{n}\left(u_{2}, v_{1}\right) \geq Q_{n+1}-\left(a_{n+1}-u_{2}\right)-$ $\left(b_{n+1}-v_{1}\right)$, then $V_{B_{n+1}}(R) \geq B_{n}\left(u_{1}, v_{1}\right)+B_{n}\left(u_{2}, v_{2}\right)-B_{n}\left(u_{1}, v_{2}\right)-B_{n}\left(u_{2}, v_{1}\right) \geq 0$ by the induction hypothesis.

- Assume that $B_{n}\left(u_{1}, v_{2}\right) \leq Q_{n+1}-\left(a_{n+1}-u_{1}\right)-\left(b_{n+1}-v_{2}\right)$. Then, by the Lipschitz property of $B_{n}$, necessarily $B_{n}\left(u_{2}, v_{2}\right) \leq Q_{n+1}-\left(a_{n+1}-u_{2}\right)-\left(b_{n+1}-v_{2}\right)$, and, therefore, by the Lipschitz property of $B_{n+1}, V_{B_{n+1}}(R)=u_{2}-u_{1}+B_{n}\left(u_{1}, v_{1}\right)-$ $B_{n}\left(u_{2}, v_{1}\right) \geq 0$.

- The remaining case, when $B_{n}\left(u_{1}, v_{2}\right) \geq Q_{n+1}-\left(a_{n+1}-u_{1}\right)-\left(b_{n+1}-v_{2}\right)$ and $B_{n}\left(u_{2}, v_{1}\right) \leq Q_{n+1}-\left(a_{n+1}-u_{2}\right)-\left(b_{n+1}-v_{1}\right)$, is treated similarly to the second case.

Proof of Theorem 2. We give the proof for the bound $\tilde{A}^{r}(u, v)$. Since the proof is based only on Proposition 1, which holds in the same form both for copulas and for quasi-copulas, $A^{r}$ coincides with $\tilde{A}^{r}$. The proofs for the lower bounds $\tilde{B}^{r}$ and $B^{r}$ are similar.

Assume that $r \in\left[\rho(W), \rho_{-}(u, v, M(u, v))\right]$. Then, since $\theta \mapsto \rho_{-}(u, v, \theta)$ is increasing and continuous,

$$
\rho\left(C_{L}^{u, v, \rho_{-}^{-1}(u, v, r)}\right)=r
$$

and, therefore, $A^{r}(u, v) \geq \rho_{-}^{-1}(u, v, r)$. On the other hand,

$$
\{\rho(Q) \mid Q(u, v)=\theta\} \subseteq\left[\rho_{-}(u, v, \theta), \rho_{+}(u, v, \theta)\right] .
$$

By the definition of $\rho_{-}^{-1}$, for all $\theta>\rho_{-}^{-1}(u, v, r), \rho_{-}(u, v, \theta)>r$ and, therefore, for every $Q \in \mathcal{Q}$ such that $Q(u, v)>\rho_{-}^{-1}(u, v, r), \rho(Q)>r$. Therefore, $A^{r}(u, v) \leq \rho_{-}^{-1}(u, v, r)$.

Assume now that $r>\rho_{-}(u, v, M(u, v))$, and let $C^{w}:=(1-w) C_{L}^{u, v, M(u, v)}+w M(u, v)$. Then $\rho\left(C^{0}\right)<r, \rho\left(C^{1}\right) \geq r$ (by the assumption of the theorem), and since $\rho$ is continuous, there exists $w \in[0,1]$ such that $\rho\left(C^{w}\right)=r$. Since $C^{w}(u, v)=M(u, v)$ for all $w$, this proves that $A^{r}(u, v) \geq M(u, v)$. On the other hand, clearly, $A^{r}(u, v) \leq M(u, v)$ (Fréchet bound). 
Proof of Proposition 2. Since $f$ is 2-increasing, $V_{f}([0, x] \times[0, y])=f(x, y)+f(0,0)-$ $f(x, 0)-f(0, y)$ is increasing in $x$ and $y$, and, therefore,

$$
\begin{aligned}
|f(x, y)| & =\left|V_{f}([0, x] \times[0, y])-f(0,0)+f(x, 0)+f(0, y)\right| \\
\leq & |f(0,0)|+|f(x, 0)|+|f(0, y)|+\left|V_{f}\left([0, x]^{2}\right)\right|+\left|V_{f}\left([0, y]^{2}\right)\right| \\
\leq & C\{|f(0,0)|+|f(0, x)|+|f(x, 0)|+|f(0, y)|+|f(y, 0)|+|f(x, x)| \\
& \quad+|f(y, y)|\}
\end{aligned}
$$

for some $C>0$, which implies that $\mathrm{E}[|f(X, Y)|]<\infty$.

Let $p(\mathrm{~d} x \times \mathrm{d} y)$ be the law of $(X, Y)$. By Fubini's theorem and (7) we then get

$$
\begin{aligned}
\pi(C)= & \mathrm{E}[f(X, Y)] \\
= & -f(0,0)+\mathrm{E}[f(X, 0)]+\mathrm{E}[f(0, Y)] \\
& +\int_{0}^{\infty} \int_{0}^{\infty} \int_{0}^{\infty} \int_{0}^{\infty} \mathbf{1}_{\left\{x^{\prime}<x\right\}} \mathbf{1}_{\left\{y^{\prime}<y\right\}} \mu\left(\mathrm{d} x^{\prime} \times \mathrm{d} y^{\prime}\right) p(\mathrm{~d} x \times \mathrm{d} y) \\
= & -f(0,0)+\mathrm{E}[f(X, 0)]+\mathrm{E}[f(0, Y)] \\
& +\int_{0}^{\infty} \int_{0}^{\infty} \mu\left(\mathrm{d} x^{\prime} \times \mathrm{d} y^{\prime}\right) \mathrm{P}\left[X>x^{\prime}, Y>y^{\prime}\right] \\
= & -f(0,0)+\mathrm{E}[f(X, 0)]+\mathrm{E}[f(0, Y)] \\
& +\int_{0}^{\infty} \int_{0}^{\infty} \mu(\mathrm{d} x \times \mathrm{d} y)\left(1-F_{X}(x)-F_{Y}(y)+C\left(F_{X}(x), F_{Y}(Y)\right)\right) .
\end{aligned}
$$

In the last integral, the integrand is positive and bounded from above by the function $1-F_{X}(x)-$ $F_{Y}(y)+\min \left\{F_{X}(x), F_{Y}(y)\right\}$, which corresponds to the copula of complete dependence and is integrable by the first part of the proposition. Therefore, the dominated convergence theorem implies that $\pi(C)$ is continuous with respect to pointwise convergence of copulas.

\section{Acknowledgements}

This research was supported by the Chair Financial Risks of the Risk Foundation sponsored by the Société Générale, the Chair Derivatives of the Future sponsored by the Fédération Bancaire Française, and the Chair Finance and Sustainable Development sponsored by EDF and Calyon.

The author would like to thank K. Jean-Alphonse for numerous discussions which helped to shape the paper and for doing preliminary simulations for this research during his internship at École Polytechnique. Thanks are also due to the anonymous referee whose comments helped to improve the presentation of the paper.

\section{References}

[1] Dhaene, J. and Goovaerts, M. (1996). Dependency of risks and stop-loss order. ASTIN Bull. 26, 201-212.

[2] Fredricks, G. A. And Nelsen, R. B. (2002). The Bertino family of copulas. In Distributions with Given Marginals and Statistical Modelling, Kluwer, Dordrecht, pp. 81-92.

[3] Genest, C., Quesada-Molina, J. J., Rodriguez Lallena, J. A. And SemPi, C. (1999). A characterization of quasi-copulas. J. Multivariate Anal. 69, 193-205.

[4] Hobson, D., Laurence, P. and Wang, T.-H. (2005). Static-arbitrage optimal subreplicating strategies for basket options. Insurance Math. Econom. 37, 553-572.

[5] Hobson, D., Laurence, P. And Wang, T.-H. (2005). Static-arbitrage upper bounds for the prices of basket options. Quant. Finance 5, 329-342. 
[6] KaAs, R., LAeven, R. J. A. And Nelsen, R. B. (2009). Worst VaR scenarios with given marginals and measures of association. Insurance Math. Econom. 44, 146-158.

[7] Kingman, J. F. C. AND TAYlor, S. J. (1966). Introduction to Measure and Probability. Cambridge University Press.

[8] Müller, A. ANd Scarsini, M. (2000). Some remarks on the supermodular order. J. Multivariate Anal. 73, $107-119$.

[9] Nelsen, R. B. (2006). An Introduction to Copulas, 2nd edn. Springer, New York.

[10] Nelsen, R. B., Quesada-Molina, J. J., Rodriguez Lallena, J. A. And Úbeda-Flores, M. (2001). Bounds on bivariate distribution functions with given margins and measures of associations. Commun. Statist. Theory Meth. 30, 1155-1162.

[11] Nelsen, R. B., Quesada-Molina, J. J., Rodriguez Lallena, J. A. and Úbeda-Flores, M. (2004). Bestpossible bounds on sets of bivariate distribution functions. J. Multivariate Anal. 90, 348-358.

[12] Rapuch, G. AND Roncalli, T. (2001). Some remarks on two-asset options pricing and stochastic dependence of asset prices. Tech. Rep., Groupe de Recherche Operationnelle, Credit Lyonnais.

[13] Tchen, A. H. (1980). Inequalities for distributions with given margins. Ann. Appl. Prob. 8, 814-827. 